POS $\quad$ PROCEEDINGS

\title{
Wilson chiral perturbation theory for $2+1$ flavors
}

\section{Sinya Aoki}

Graduate School of Pure and Applied Sciences, University of Tsukuba, Tsukuba, Japan

E-mail: saoki@het.ph.tsukuba.ac.jp

\section{Oliver Bär}

Graduate School of Pure and Applied Sciences, University of Tsukuba, Tsukuba, Japan

E-mail: obaer@het.ph.tsukuba.ac.jp

\section{Tomomi Ishikawa}

Center for Computational Sciences, University of Tsukuba, Tsukuba, Japan

E-mail: tomomi@ccs.tsukuba.ac.jp

\section{Shinji Takeda* ${ }^{\dagger}$}

Graduate School of Pure and Applied Sciences, University of Tsukuba, Tsukuba, Japan

E-mail: takeda@het.ph.tsukuba.ac.jp

We consider $2+1$ flavor Wilson chiral perturbation theory including the lattice spacing contributions of $\mathrm{O}\left(a^{2}\right)$. We adopt a power counting which the $\mathrm{O}\left(a^{2}\right)$ terms are included in a leading order. Here, we present a result of the pseudo scalar meson masses to one loop. The mass formulae are required to perform the chiral extrapolation of the CP-PACS/JLQCD lattice data.

XXIIIrd International Symposium on Lattice Field Theory

25-30 July 2005

Trinity College, Dublin, Ireland

\footnotetext{
${ }^{*}$ Speaker.

$\dagger$ The author is supported by Research Fellowships of the Japan Society for the Promotion of Science for Young Scientists (Nos. 16.11968).
} 


\section{Introduction}

The development of the algorithm [1] and current powerful machines [2] allows us to perform unquenched $2+1$ flavor simulation whose results can be compared with experiment with confidence. CP-PACS and JLQCD collaborations have been carrying out the unquenched $2+1$ flavor simulations where the Iwasaki gauge action and an $\mathrm{O}(a)$ improved Wilson quark action have been adopted. Five different masses for the degenerate up and down type quarks are simulated leading to pseudo scalar meson masses in the range $m_{P S} / m_{V} \simeq 0.62-0.78$. The physical strange quark mass lies between the two simulated strange quark masses and can therefore be reached by an interpolation. More details and the status of these simulations have been reported in the proceedings by T. Ishikawa.

The masses for the up and down quarks are rather heavy and an extrapolation to their physical values is required. In order to achieve the extrapolation in a reliable way, we derive the functional forms of physical observables on the basis of the Wilson chiral perturbation theory(WChPT). The theory is formulated for lattice QCD with Wilson type fermions at non-zero lattice spacing $a$. The main idea how this can be done was proposed in Ref. [3, 4]. Since then many observables have been calculated at one loop order (for an overview see Ref. [5]).

Here we present the results for the simplest observables, the pseudo scalar meson masses. In our calculations we include the $\mathrm{O}\left(a^{2}\right)$ terms in the leading order Lagrangian. We refer to ref. [6] for details and unexplained notations in the report. The other observables(vector meson masses, the pseudo scalar decay constants and axial vector Ward identity quark masses) can be computed in a straightforward way and will be shown in other places[7,8].

\section{Counting scheme}

First of all, we have to specify an order counting scheme for an effective Lagrangian. The leading order (LO) chiral Lagrangian of the WChPT is constructed from $\mathrm{O}(M)$ terms and the $\mathrm{O}(a)$ term. Since the $\mathrm{O}(a)$ term has the same chiral structure as the mass term, the LO Lagrangian of the WChPT assumes the same form as the one of continuum ChPT, provided one performs the replacement $m \rightarrow \tilde{m}=m+c_{1} a$ with $c_{1}$ being a combination of two low-energy constants [3,9]. Although the $\mathrm{O}(a)$ term is superficially larger than the $\mathrm{O}\left(a^{2}\right)$ term, the former term is irrelevant since it is absorbed in the definition of the shifted mass $\tilde{m}$. The $\mathrm{O}\left(a^{2}\right)$ correction, on the other hand, becomes important in the regime where $\tilde{m} / \Lambda_{\mathrm{QCD}} \simeq \Lambda_{\mathrm{QCD}}^{2} a^{2}$, even though the $\Lambda_{\mathrm{QCD}}^{2} a^{2}$ correction is much smaller than 1 in general.

Suppose we consider the $\mathrm{O}(\tilde{M})=O\left(\tilde{m}, p^{2}\right)$ and the $\mathrm{O}\left(a^{2}\right)$ term as LO terms. Then the terms of $\mathrm{O}\left(\tilde{M}^{2}, \tilde{M} a^{2}, a^{4}\right)$ can be regarded as next to leading order (NLO), since the loop expansion in WChPT increases in units of $\tilde{M}$. We remark that the $\mathrm{O}\left(a^{4}\right)$ term is not as relevant as the $\mathrm{O}\left(\tilde{M}^{2}, \tilde{M} a^{2}\right)$ terms for our final results for the pseudo scalar masses.

The proper order counting of the $\mathrm{O}(\tilde{M} a)$ term is more subtle than for the previously discussed terms. ${ }^{1}$ Depending on the size of the $\mathrm{O}(\tilde{M} a)$ contributions we may include it at leading order where it subsequently enters the chiral logs, or we treat it as a NLO term and include it at tree level

\footnotetext{
${ }^{1}$ The $\mathrm{O}\left(a^{3}\right)$ term is unproblematic since the arguments we gave for the $\mathrm{O}\left(a^{4}\right)$ contribution also apply for the $\mathrm{O}\left(a^{3}\right)$ term.
} 
only. The size of this term is indeed expected to vary significantly, depending on details of the action of the underlying lattice theory. The $\mathrm{O}(\tilde{M} a)$ term contains one power of $a$ and stems from the Pauli term in the Symanzik's effective action [10,11, 12], which is used in an intermediate step to match the lattice to the chiral effective theory. Consequently, the $\mathrm{O}(\tilde{M} a)$ contribution in the chiral Lagrangian is directly proportional to the coefficient of the Pauli term [9], and the size of this coefficient is much smaller for improved theories with a clover term in the action than for standard Wilson fermions. For fully non-perturbatively improved theories the coefficient is equal to zero and no $\mathrm{O}(\tilde{M} a)$ term appears in the chiral Lagrangian.

Since we have no a priori knowledge about the size of the $\mathrm{O}(\tilde{M} a)$ term we will be as general as possible and present our results for three different order countings of this term. We first keep the $\mathrm{O}(\tilde{M} a)$ term at $\mathrm{LO}$ where it gives a contribution to the chiral log corrections. If the $\mathrm{O}(\tilde{M} a)$ term is assumed to be much smaller than the other LO terms we can easily expand our results. Performing this expansion is equivalent to doing the calculation with keeping the $\mathrm{O}(\tilde{M} a)$ term at NLO. Finally we can set this term to zero in order to obtain the results for non-perturbatively improved theories.

\section{Effective Lagrangian}

According to the spurion analysis, we find the LO Lagrangian, which consists of terms of $\mathrm{O}\left(\tilde{M}, a^{2}, \tilde{M} a\right)$ :

$$
\begin{aligned}
L_{\mathrm{LO}} & =\frac{f^{2}}{4}\left\langle\partial_{\mu} \Sigma \partial_{\mu} \Sigma^{\dagger}\right\rangle-\frac{f^{2} B}{2}\left\langle M_{q} \Sigma+\Sigma^{\dagger} M_{q}\right\rangle \\
& +\frac{f^{2}}{16}\left[c_{2}\left\langle\Sigma+\Sigma^{\dagger}\right\rangle^{2}+\tilde{c}_{2}\left\langle\left(\Sigma+\Sigma^{\dagger}\right)^{2}\right\rangle+c_{4}\left\langle\Sigma-\Sigma^{\dagger}\right\rangle^{2}\right] \\
& +\frac{f^{2}}{4}\left[c_{0}\left\langle\Sigma+\Sigma^{\dagger}-2\right\rangle\left\langle\partial_{\mu} \Sigma \partial_{\mu} \Sigma^{\dagger}\right\rangle+\tilde{c}_{0}\left\langle\left(\Sigma+\Sigma^{\dagger}-2\right) \partial_{\mu} \Sigma \partial_{\mu} \Sigma^{\dagger}\right\rangle\right] \\
& +\frac{f^{2} B}{8}\left[2 c_{3}\left\langle\Sigma+\Sigma^{\dagger}\right\rangle\left\langle M_{q} \Sigma+\Sigma^{\dagger} M_{q}\right\rangle+\tilde{c}_{3}\left\langle\left(\Sigma+\Sigma^{\dagger}\right)\left(M_{q} \Sigma+\Sigma^{\dagger} M_{q}\right)\right\rangle\right] \\
& +\frac{f^{2} B}{8}\left[2 c_{5}\left\langle\Sigma-\Sigma^{\dagger}\right\rangle\left\langle M_{q} \Sigma-\Sigma^{\dagger} M_{q}\right\rangle+\tilde{c}_{5}\left\langle\left(\Sigma-\Sigma^{\dagger}\right)\left(M_{q} \Sigma-\Sigma^{\dagger} M_{q}\right)\right\rangle\right],
\end{aligned}
$$

where $\langle X\rangle=\operatorname{tr} X . \Sigma=\exp \left[i \frac{1}{f} \sum_{a} \pi_{a} T^{a}\right]$ is an element of $\mathrm{SU}(3)$ with $\pi_{a}$ being the pseudo scalar meson fields. The SU(3) generators $T^{a}$ are normalized according to tr $T^{a} T^{b}=\frac{1}{2} \delta_{a b}$. The first and the second term in the first line are the standard $\mathrm{O}\left(p^{2}\right)$ and $\mathrm{O}(\tilde{m})$ terms [13], respectively. The second line comprises the $\mathrm{O}\left(a^{2}\right)$ terms $[14,15]$. The last three lines contain the $\mathrm{O}\left(p^{2} a\right)$ and $\mathrm{O}(\tilde{m} a)$ contributions [9].

For notational simplicity only we use a different notation for the low energy constants associated with the non-zero lattice spacing (the $c$ and $\tilde{c}$ 's) compared to the notation in Refs. [9, 14, 15]. In particular, we have chosen to absorb the explicit powers of the lattice spacing $a$ into the coefficients $c, \tilde{c}$. Consequently, as a function of $a$ these coefficients scale according to $c_{0}, \tilde{c}_{0}, c_{3}, \tilde{c}_{3}, c_{5}, \tilde{c}_{5}=\mathrm{O}(a)$ and $c_{2}, \tilde{c}_{2}, c_{4}=\mathrm{O}\left(a^{2}\right)$. The quark mass matrix is given by $M_{q}=\operatorname{diag}\left(m, m, m_{s}\right)$, where isospin symmetry is assumed. Note that $\mathrm{O}(a)$ contribution is already absorbed in the definition of $M_{q}$, so there is no $\mathrm{O}(a)$ term in the chiral Lagrangian.

The NLO Lagrangian provides the necessary counter terms in order to remove the UV divergences in 1-loop integrals. We consider $\mathrm{O}\left(\tilde{M}^{2}\right), \mathrm{O}\left(a^{2} \tilde{M}\right)$ and $\mathrm{O}\left(a \tilde{M}^{2}\right)$ terms as the next leading 
order. The first is a continuum one associated with the Gasser-Leutwyler coefficients. The complete Lagrangian at $\mathrm{O}\left(a^{2} \tilde{M}\right)$ and $\mathrm{O}\left(a \tilde{M}^{2}\right)$ is cumbersome and has not been computed so far. In our calculation, we only list the terms that contribute to the meson propagators, which we need for the calculation of the pseudo scalar masses. These terms are straightforwardly found by a standard spurion analysis and can be found in Ref. [6].

\section{Pseudo scalar meson mass formulae}

The calculation of the pseudo scalar masses from the chiral Lagrangian in the previous section is straightforward. Here we simply quote the final result for the quark mass and lattice spacing dependence of the pseudo scalar meson masses. The total contribution from LO tree, LO 1-loop plus NLO tree for $\phi=\pi, K$ and $\eta$ are given as follows:

$$
\begin{aligned}
m_{N L O, \phi}^{2} & =\left(x+P_{\phi} y\right)+\frac{1}{f^{2}}\left[\sum_{\psi=\pi, K, \eta} L_{\psi}\left(A_{\psi}^{\phi} x+B_{\psi}^{\phi} y+Q_{\psi}^{\phi} C\right)\right. \\
& \left.-\left\{E_{x} x+P_{\phi} E_{y} y+E_{x x} x^{2}+E_{y y}^{\phi} y^{2}+P_{\phi} E_{x y} x y\right\}\right]
\end{aligned}
$$

where the first parenthesis is the tree level contributions, the terms proportional to $L_{\psi}$ is the chiral logarithm contributions, and the polynomial terms from the NLO Lagrangian are in the second line. Here we have defined the $x$ and $y$,

$$
x=\frac{2 B_{0}}{3}\left(2 \tilde{m}+\tilde{m}_{\mathrm{s}}\right), \quad y=\frac{B_{0}}{3}\left(\tilde{m}-\tilde{m}_{\mathrm{s}}\right)
$$

where $\tilde{m}$ and $\tilde{m}_{\mathrm{s}}$ are the shifted mass defined in [6]. Since we are dealing with $2+1$ flavor theory, we have two independent quark masses. Here we choose the average of the quark masses $x$, and the magnitude of the $S U(3)$ flavor symmetry breaking $y$ as independent variables. The leading order pseudo scalar masses are written in a very simple form in terms of $x$ and $y$,

$$
\tilde{m}_{\pi}^{2}=x+2 y, \quad \tilde{m}_{K}^{2}=x-y, \quad \tilde{m}_{\eta}^{2}=x-2 y
$$

The chiral logarithms for each virtual pseudo-scalar meson are given as $L_{\phi}=\frac{\tilde{m}_{\phi}^{2}}{16 \pi^{2}} \ln \left(\frac{\tilde{m}_{\phi}^{2}}{\mu^{2}}\right)$,

where the $\mu$ is the renormalization scale. The numerical valued vector $P$ and matrix $Q$ are given by

$$
P_{\phi}=\left(\begin{array}{c}
P_{\pi} \\
P_{K} \\
P_{\eta}
\end{array}\right)=\left(\begin{array}{c}
2 \\
-1 \\
-2
\end{array}\right), \quad Q_{\psi}^{\phi}=\left(\begin{array}{ccc}
Q_{\pi}^{\pi} & Q_{K}^{\pi} & Q_{\eta}^{\pi} \\
Q_{\pi}^{K} & Q_{K}^{K} & Q_{\eta}^{K} \\
Q_{\pi}^{\eta} & Q_{K}^{\eta} & Q_{\eta}^{\eta}
\end{array}\right)=\left(\begin{array}{lll}
5 & 4 & 1 \\
3 & 6 & 1 \\
3 & 4 & 3
\end{array}\right) .
$$

The coefficients of the chiral logarithms, $A_{\psi}^{\phi}, B_{\psi}^{\phi}$ and $C$ are functions of the low energy constants in LO Lagrangian, $c_{i}$ and $\tilde{c}_{i}$. The $A_{\psi}^{\phi}$ and $B_{\psi}^{\phi}$ have typical form as a function of the lattice spacing $a$,

$$
A_{\psi}^{\phi}, B_{\psi}^{\phi}=w_{0}+w_{1} a+O\left(a^{2}\right),
$$

where $w_{0}$ is the continuum value and $w_{1} a$ is a linear combination of $c_{0}, \tilde{c}_{0}, c_{3}, \tilde{c}_{35}$ and $c_{5}$. The $C$ is proportional to $a^{2}$. For the coefficients of the NLO polynomial terms, the $E_{x}$ and $E_{y}$, whose origin 
is $\mathrm{O}\left(a^{2} \tilde{M}\right)$ terms, scale according to $a^{2}$ and vanish in the continuum limit, whereas, the $E_{x x}, E_{y y}^{\phi}$ and $E_{x y}$ have typical form,

$$
E=v_{0}+v_{1} a+O\left(a^{2}\right)
$$

where $v_{0}$ are some linear combination of the Gasser-Luetwyler coefficients and $v_{1} a$ are functions of the low energy constants of $\mathrm{O}\left(a \tilde{M}^{2}\right)$ terms. In order to cancel the divergence from the loop integral, the low energy constants in the polynomial terms need to be renormalized.

The $A_{\psi}^{\phi}$ and $B_{\psi}^{\phi}$ terms in eq.(4.1) produce the chiral logarithms of type $a \tilde{m}_{\mathrm{PS}}^{4} \ln \tilde{m}_{\mathrm{PS}}^{2}$. The origin of this logarithm are $O\left(a \tilde{M}^{2}\right)$ terms in the LO Lagrangian. Furthermore, $C$ term in eq.(4.1) produces the more chirally singular logarithm of type $a^{2} \tilde{m}_{\mathrm{PS}}^{2} \ln \tilde{m}_{\mathrm{PS}}^{2}$, whose origin is $O\left(a^{2}\right)$ terms in the LO Lagrangian. These kinds of logarithms were already observed in $N_{\mathrm{f}}=2$ case in Ref.[15]. We have confirmed the behavior also in $N_{\mathrm{f}}=2+1$ case.

Now, our main purpose is to perform fits for the pseudo-scalar meson masses. It is practically important to check the number of independent fit parameters. We find that the independent fit parameters are $f, B_{0}, K_{c}, c_{0}, \tilde{c}_{0}, c_{3} \tilde{c}_{35}, c_{5}, C, E_{x}, E_{y}, E_{x x}, E_{y y}^{\pi}, E_{y y}^{K}, E_{y y}^{\eta}$ and $E_{x y}$, and the total number is 16 . The number is larger than that of the conventional polynomial fit used in the [16] where the number is eight.

In eq. (4.1), $\mathrm{O}(\tilde{M} a)$ terms are considered at LO. Here let us see the case that these terms are treated as NLO corrections and we call the counting scheme WChPT scheme B (We call the original one WChPT scheme A). It is natural to image that the scheme A is applicable to coarse lattices than the scheme B. On the other hand, the scheme B has an advantage that the number of fit parameters are smaller than that of the scheme A. In order to see this point, let us check formulae for the scheme B. Forms with the scheme B are basically same as the case of scheme A in eq.(4.1), but with $w_{1}=0$ and $v_{1}=0$ in eqs. (4.5) and (4.6). The equation, $w_{1}=0$, indicates the absence of the chiral logarithms of type, $a \tilde{m}_{\mathrm{PS}}^{4} \ln \tilde{m}_{\mathrm{PS}}^{2}$. However, the more chirally singular one, $a^{2} \tilde{m}_{\mathrm{PS}}^{2} \ln \tilde{m}_{\mathrm{PS}}^{2}$, remains in the mass formulae in the scheme B. One should keep in mind that, the $E_{x}$ and $E_{y}$ start at $O(a)$ in the scheme $\mathrm{B}$, since now $O(a \tilde{M})$ terms are in the NLO Lagrangian, although they are $O\left(a^{2}\right)$ in the scheme A. The scaling order is different depending on the counting scheme. Note that $E_{x x}, E_{y y}^{\pi}, E_{y y}^{K}$ and $E_{x y}$ are not independent any more(see [6] for more details). The number of independent parameters is reduced compared to the result given in the previous section. We find that the total number of independent parameters is reduced from 16 to 9.

In the actual simulation for $2+1$ flavors by CP-PACS/JLQCD collaborations, the non-perturbative $O(a)$ improved Wilson quark action are employed. In this case, it is possible to reduce of the number of fit parameter. With the above action (and non-perturbative $O(a)$ improved composite operators), there are no $O(a)$ terms in one-shell quantities. This means that there exists no Pauli term in the Symanzik's effective theory, but with replacements

$$
\begin{aligned}
m & \longrightarrow m+b_{m}^{(1)} m^{2} a+b_{m}^{(2)}\left(2 m+m_{\mathrm{s}}\right) m a+b_{m}^{(3)}\left(2 m^{2}+m_{\mathrm{s}}^{2}\right) a \\
m_{\mathrm{s}} & \longrightarrow m_{\mathrm{s}}+b_{m}^{(1)} m_{\mathrm{s}}^{2} a+b_{m}^{(2)}\left(2 m+m_{\mathrm{s}}\right) m_{\mathrm{s}} a+b_{m}^{(3)}\left(2 m^{2}+m_{\mathrm{s}}^{2}\right) a \\
g_{0}^{2} & \longrightarrow g_{0}^{2}\left(1+b_{g} \frac{\left(2 m+m_{\mathrm{s}}\right) a}{3}\right) .
\end{aligned}
$$

As a consequence, as long as one considers on-shell quantities, $O(a), O(a \tilde{M}), O\left(a \tilde{M}^{2}\right), \cdots$ terms 
are absent in the WChPT, but with replacement

$$
\begin{aligned}
\tilde{m} \longrightarrow \bar{m} & =\tilde{m}+b_{m}^{(1)} \tilde{m}^{2} a+b_{m}^{(2)}\left(2 \tilde{m}+\tilde{m}_{\mathrm{s}}\right) \tilde{m} a+b_{m}^{(3)}\left(2 \tilde{m}^{2}+\tilde{m}_{\mathrm{s}}^{2}\right) a \\
\tilde{m}_{\mathrm{s}} \longrightarrow \bar{m}_{\mathrm{s}} & =\tilde{m}_{\mathrm{s}}+b_{m}^{(1)} \tilde{m}_{\mathrm{s}}^{2} a+b_{m}^{(2)}\left(2 \tilde{m}+\tilde{m}_{\mathrm{s}}\right) \tilde{m}_{\mathrm{s}} a+b_{m}^{(3)}\left(2 \tilde{m}^{2}+\tilde{m}_{\mathrm{s}}^{2}\right) a \\
B_{0} \longrightarrow \bar{B}_{0} & =B_{0}\left[1+b_{B}\left(2 \tilde{m}+\tilde{m}_{\mathrm{s}}\right) a\right] .
\end{aligned}
$$

The last modification comes from the mass dependence of $g_{0}^{2}$ (eq.(4.9)) in the Symanzik's effective theory. In this case, it turns out that the structure of the chiral logs are the same as WChPT scheme $\mathrm{B}$ case The number of independent parameters is 10, reduced from previously found 16 .

\section{Concluding remarks}

In the report we computed the pseudo scalar meson masses in $2+1$ flavor WChPT to 1-loop order. We considered results for three different order countings, appropriate for various sizes of the $\mathrm{O}(a \tilde{M})$ term in the chiral Lagrangian. Depending on the lattice action used in the numerical simulation (unimproved, perturbatively improved, non-perturbatively improved) one has to choose one result for the chiral extrapolation. Since we have no prior knowledge about the size of the $\mathrm{O}(a \tilde{M})$ contribution we suggest to perform fits to the data with all three forms and let the data decide which form is most appropriate. The number of unknown fit parameters is significantly larger than in 2 flavor WChPT. Performing the chiral extrapolation of the CP-PACS/JLQCD data using our results is work in progress.

\section{References}

[1] S. Aoki et al., Phys. Rev. D65 (2002) 094507.

[2] S. Aoki et al., Nucl. Phys. Proc. Suppl. 129 (2004) 859.

[3] S. R. Sharpe and R. Singleton Jr., Phys. Rev. D58 (1998) 074501.

[4] W.-J. Lee and S. R. Sharpe, Phys. Rev. D60 (1999) 114503.

[5] O. Bär, hep-lat/0409123.

[6] S. Aoki, O. Bär, T. Ishikawa and S. Takeda, hep-lat/0509049.

[7] S. Aoki, O. Bär and S. Takeda, Vector meson masses in $2+1$ flavor Wilson Chiral Perturbation Theory, in preparation.

[8] S. Aoki, O. Bär and S. Takeda, Pseudo scalar decay constants in 2+1 flavor Wilson Chiral Perturbation Theory, work in progress.

[9] G. Rupak and N. Shoresh, Phys. Rev. D66 (2002) 054503.

[10] K. Symanzik, Nucl. Phys. B226 (1983) 187.

[11] K. Symanzik, Nucl. Phys. B226 (1983) 205.

[12] B. Sheikholeslami and R. Wohlert, Nucl. Phys. B259 (1985) 572.

[13] J. Gasser and H. Leutwyler, Nucl. Phys. B250 (1985) 465.

[14] O. Bär, G. Rupak and N. Shoresh, Phys. Rev. D70 (2004) 034508.

[15] S. Aoki, Phys. Rev. D68 (2003) 054508.

[16] T. Ishikawa et al., hep-lat/0409124. 